\title{
A RARE CASE OF TORSION OF WANDERING SPLEEN IN A MULTIPAROUS WOMAN
}

S. Venkateshwar Rao ${ }^{1}$, Shirish Paul Ganta², N. Kiran Raju³, V. N. Narvekar, V. Sivaleela5

\section{HOW TO CITE THIS ARTICLE:}

S. Venkateshwar Rao, Shirish Paul Ganta, N. Kiran Raju, V. N. Narvekar, V. Sivaleela. "A Rare Case of Torsion of Wandering Spleen in a Multiparous Woman". Journal of Evolution of Medical and Dental Sciences 2015; Vol. 4, Issue 12, February 09; Page: 2082-2086, DOI: 10.14260/jemds/2015/299

ABSTRACT: Wandering spleen is a rare clinical entity characterized by splenic hypermobility resulting from laxity or maldevelopment of supporting splenic ligaments ${ }^{1}$. Its major complication is splenic torsion, which is a potentially fatal surgical emergency ${ }^{2}$. We present a rare case of wandering spleen with torsion and splenic infarction in a multiparous patient.

KEYWORDS: Wandering spleen, hypogastrium, pelvis, ultrasound, computed tomography, MRI.

CASE REPORT: A 52- year old female presented with abdominal pain for 10 days. On clinical examination the patient was afebrile, moderately built and fully conscious. There was no jaundice, cyanosis or lymphadenopathy. Abdominal examination revealed that the abdomen was soft with no organomegaly. Splenic fossa was empty. There was an approximately $17 x 7 \mathrm{~cm}$ sized mass palpable in the hypogastrium, extending into the pelvis, which was firm and sharp in consistency and slightly tender and non-mobile in nature. The inferior margin of this mass could not be appreciated and was dull on percussion. Per-vaginal examination showed that there were no adnexal mass lesions. Examinations of the cardiovascular and central nervous systems were unremarkable. The patient had a heart rate of 80 beats per minute, a respiratory rate of 16 breaths per minute and blood pressure of 114/70 mmHg. Liver (serum glutamic oxaloacetic transaminase, serum glutamic pyruvic transaminase, bilirubin) and renal (urea and creatinine) function tests were within normal limits. A full blood counts were normal.

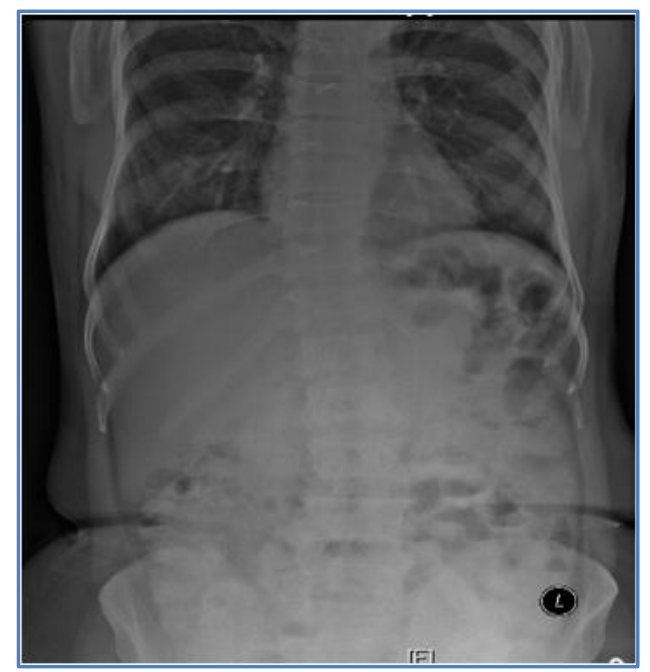

Fig. 1: Plain radiograph was unremarkable 


\section{CASE REPORT}

Ultrasound of the abdomen indicated the absence of a normal spleen in the left hypochondrium (Fig. 2) and the presence of a sickle-shaped mass $(18 \mathrm{x} 9 \mathrm{~cm})$ with echotexture similar to that of spleen in the hypogastrium and pelvis (Fig. 3), superior to the bladder. Its echotexture was relatively hypoechoic when compared with the rest of the splenic parenchyma and colour doppler revealed absent colour flow at hilum. These findings were suspicious of torsion of wandering spleen with infarction. The liver, gall bladder, both kidneys and pancreas were normal.

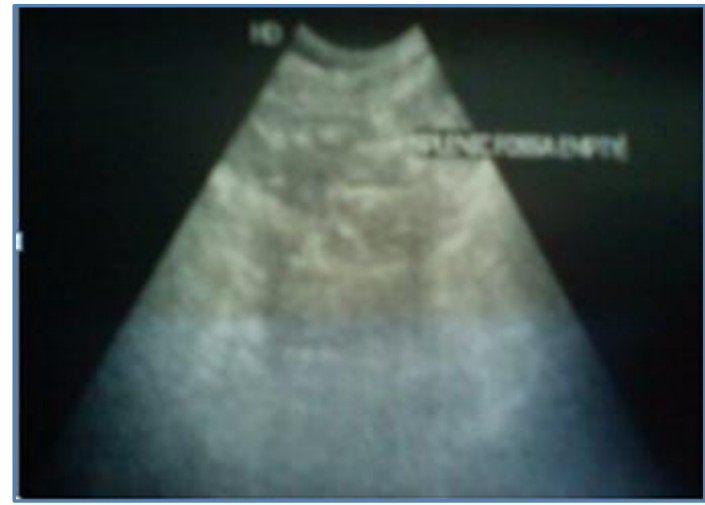

Fig. 2: Splenic fossa empty

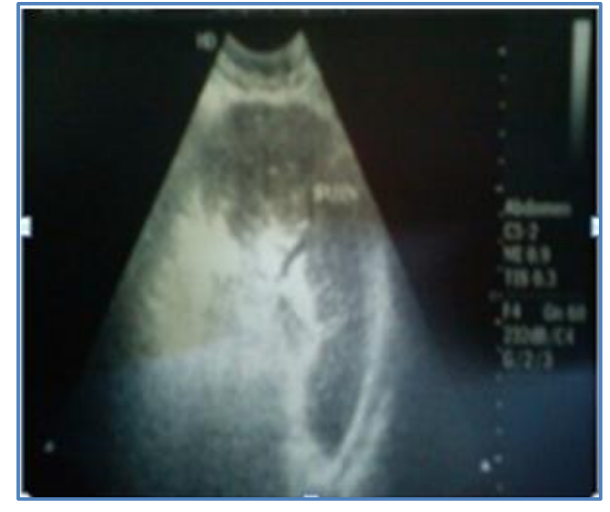

Fig. 3: Spleen in hypogastrium and pelvis

Subsequently, contrast-enhanced CT of the abdomen was performed, which confirmed the absence of spleen in the left hypochondrium and its presence in the hypogastrium and pelvis. The spleen was enlarged. The entire parenchyma was hypodense and non-enhancing, Whirl sign was positive-suggestive of splenic infarction with torsion ${ }^{3}$ (Fig. 4). No areas of calcification or haemorrhage were seen.

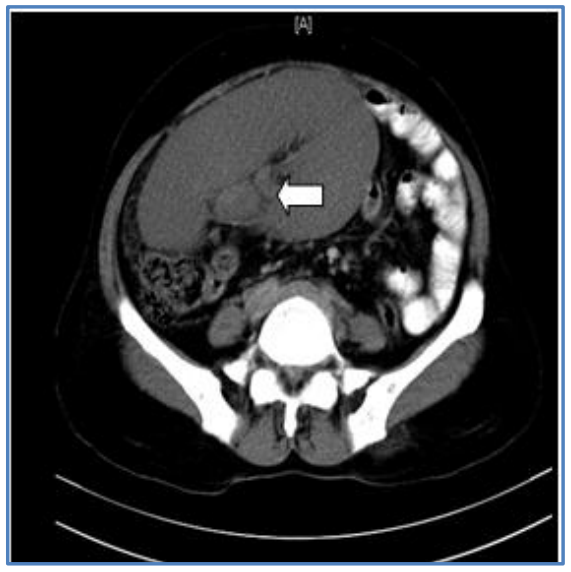

Fig. 4: CECT reveals whirl sign, a whirled appearance of hyperdense, nonenhancing splenic vessels-indicative of torsion (arrows)

Coronal MR images reveals an ovoid or comma-shaped abdominal mass in hypogastrium region. (Fig. 5) 


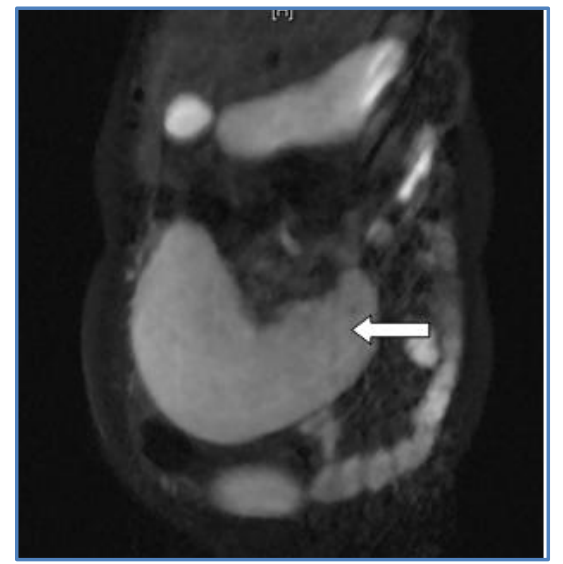

Fig. 5: Coronal MR Image ovoid or commashaped abdominal mass in hypogastrium region

DISCUSSION: The aetiology of wandering spleen is divided into a congenital form and an acquired form. The congenital form results from a lack of development of the primary ligamentous attachments of the spleen: the gastrosplenic, splenorenal, splenocolic splenophrenic, splenopancreatic, presplenic fold, pancreaticocolic, and phrenocolic ligaments ${ }^{4}$. An assumption made when hypothesizing that wandering spleen is of congenital origin is that the dorsal mesogastrium fails to fuse to the posterior abdominal wall during embryonic development.

The acquired form is believed to be caused by a laxity of these ligaments, which might be attributable to hormonal changes, splenomegaly, trauma or multiparity. Lack of ligamentous support allows the spleen to twist on its own pedicle. This torsion impairs venous return, causing congestion and capsular stretching. If torsion progresses, arterial supply is compromised, thus causing infarction, fibrosis and necrosis. Torsion complicates $64 \%$ of paediatric wandering spleen cases and the extent of torsion varies from $90^{\circ}$ to $216^{\circ}$.

The presentation of wandering spleen is varied. Patients may be (i) asymptomatic, (ii) present with a moveable lump in the abdomen, or (iii) present with acute, chronic or intermittent pain in the abdomen caused by torsion of the wandering spleen 5 .

Early clinical diagnosis is difficult, and hence imaging modalities play an important role. The amount of vascular compromise can alter the intensity of pain secondary to capsular stretching, splenic engorgement and local peritonitis. Complications of acute splenic torsion include gangrene, abscess formation, local peritonitis, intestinal obstruction and necrosis of the pancreatic tail. Recurrent acute pancreatitis has been reported as a manifestation of this condition.

Multiple imaging techniques have been used to diagnose a wandering spleen with torsion, including plain radiography, barium studies, scintigraphy, ultrasound, CT and MRI.

Conventional radiography can sometimes show the absence of a splenic shadow in the leftupper quadrant, along with a central abdominal or pelvic mass.

However, plain radiography and barium studies are usually non-specific and may, in some cases, show the medial or superior displacement with extrinsic impression on the splenic flexure of the colon, along with a soft tissue mass in the central abdomen or pelvis, corresponding to the wandering spleen. Ultrasound and CT can both demonstrate the lack of splenic tissue in the leftupper quadrant and the characteristic comma shaped enlarged spleen in an ectopic location. 


\section{CASE REPORT}

Sonography can also show involvement of neighboring structures such as the tail of the pancreas, which may be enlarged and show a changed echotexture if infarcted . However, sonography can often be hampered by bowel gas.

CT is the preferred modality for diagnosing a wandering spleen when torsion is suspected clinically or is based on imaging studies. Doppler study is useful for providing information on the splenic vasculature (parenchyma) and vascular pedicle. Doppler examination of the splenic artery distal to the bifurcation of the coeliac artery may show an increased resistive index of over $80 \%$ and low diastolic velocity in patients with acute torsion of the splenic vascular pedicle.

CT signs of a wandering spleen with acute torsion include: absence of spleen anterior to the left kidney and posterior to the stomach; a lower abdominal or pelvic mass, with homogeneous /heterogeneous splenic parenchyma and an attenuation value less than that of normal splenic tissue; whorled appearance of splenic vessels and surrounding fat; and the presence of secondary findings such as ascites and necrosis of the pancreatic tail. However, it is the whorled appearance of the splenic vessels and surrounding fat at the splenic hilum that is considered characteristic of torsion of wandering spleen.

Previously, splenectomy was the standard recommended treatment for wandering spleen, with or without the presence of torsion. However, present opinion favours treatment of a viable wandering spleen by splenopexy ${ }^{4}$, especially in paediatric patients, to avoid the risk of postsplenectomy sepsis. The vascular status of the spleen is the key determinant in deciding if the spleen can be salvaged by splenopexy. However, splenectomy is indicated if the spleen is at functional risk of rupture. Although rare, splenic torsion should be recognized as a cause of acute abdomen. An increased awareness of this condition is required, along with the timely use of imaging that can help in making a prompt and accurate diagnosis for appropriate management.

\section{REFERENCES:}

1. Walker BA, Beighton PH, Murdoch JL. The marfanoid hypermobility syndrome. Ann Intern Med 1969; 71: 349-52.

2. Sodhi KS, Saggar K, Sood BP, Sandhu P. Torsion of a wandering spleen: acute abdominal presentation. J Emerg Med 2003; 25: 133-7.

3. Taori K, Ghonge N, Prakash A. Wandering spleen with torsion of vascular pedicle: early diagnosis with multiplaner reformation technique of multislice spiral CT. Abdom Imaging 2004; 29: 479-81.

4. Allen $\mathrm{KB}$,Andrewa G. Pediatric wandering spleen-the case for splenopexy: review of 35 reported cases in the literature. J Pediatr Surg 1989;24:432

5. Romero JR, Barksdale EM Jr. Wandering spleen: a rare cause of abdominal pain. Pediatr Emerg Care 2003; 19: 412-14. 


\section{CASE REPORT}

\section{AUTHORS:}

1. S. Venkateshwar Rao

2. Shirish Paul Ganta

3. N. Kiran Raju

4. V. N. Narvekar

5. V. Sivaleela

\section{PARTICULARS OF CONTRIBUTORS:}

1. Associate Professor, Department of Radiology, Asram Medical College.

2. Assistant Professor, Department of Radiology, Asram Medical College.

3. Assistant Professor, Department of Radiology, Asram Medical College.

4. Professor and HOD, Department of Radiology, Asram Medical College.

5. Professor, Department of Radiology, Asram Medical College.

\section{NAME ADDRESS EMAIL ID OF THE} CORRESPONDING AUTHOR:

Dr. S. Venkateshwar Rao,

Associate Professor,

Department of Radiodiagnosis,

Asram Medical College,

Eluru, West Godavari Direct,

Andhra Pradesh - 534005.

E-mail: drsvrao999@gmail.com

Date of Submission: 25/12/2014.

Date of Peer Review: 26/12/2014.

Date of Acceptance: 30/01/2015.

Date of Publishing: 09/02/2015. 